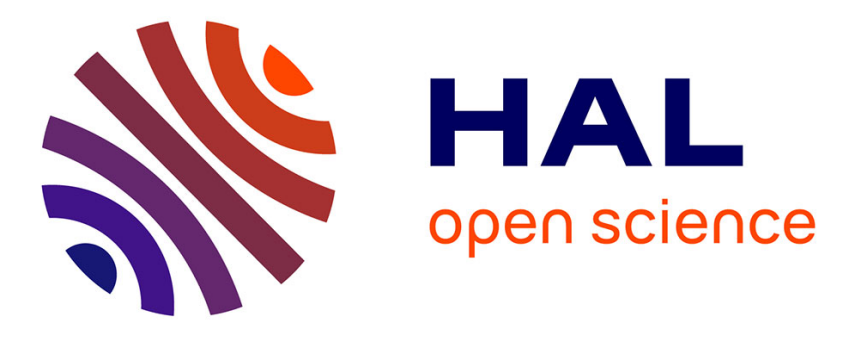

\title{
An introduction to algebraic discrete-time linear parametric identification with a concrete application
}

Michel Fliess, Stefan Fuchshumer, Markus Schöberl, Kurt Schlacher, Hebertt Sira-Ramirez

\section{- To cite this version:}

Michel Fliess, Stefan Fuchshumer, Markus Schöberl, Kurt Schlacher, Hebertt Sira-Ramirez. An introduction to algebraic discrete-time linear parametric identification with a concrete application. Journal Européen des Systèmes Automatisés (JESA), 2008, 42 (2-3), pp.210-232. inria-00188435v2

\section{HAL Id: inria-00188435 https://hal.inria.fr/inria-00188435v2}

Submitted on 9 Apr 2008

HAL is a multi-disciplinary open access archive for the deposit and dissemination of scientific research documents, whether they are published or not. The documents may come from teaching and research institutions in France or abroad, or from public or private research centers.
L'archive ouverte pluridisciplinaire HAL, est destinée au dépôt et à la diffusion de documents scientifiques de niveau recherche, publiés ou non, émanant des établissements d'enseignement et de recherche français ou étrangers, des laboratoires publics ou privés. 


\title{
An introduction to algebraic discrete-time linear parametric identification with a concrete application
}

\author{
Michel Fliess ${ }^{1,2}$ - Stefan Fuchshumer ${ }^{3,4}$ - Markus Schöberl ${ }^{4}$ - \\ Kurt Schlacher $^{4}$ - Hebertt Sira-Ramírez ${ }^{5}$
}

1 INRIA - ALIEN

2 Equipe MAX, LIX (CNRS, UMR 7161),

Ecole polytechnique, 91128 Palaiseau, France

Michel.Fliess@polytechnique.edu

3 voestalpine mechatronics $\mathrm{GmbH}$ - vatron, voestalpine Straße 3, 4031 Linz, Austria

stefan.fuchshumer@vatron.com

4 Institut für Regelungstechnik und Prozessautomatisierung, Johannes Kepler Universität Linz, Altenbergerstraße 69, 4040 Linz, Austria

Markus.Schoeberl@jku . at \& kurt.schlacher@jku.at

5 CINVESTAV-IPN, Dept. Ingeniería Eléctrica, Sección Mecatrónica, Avenida IPN, No.2508, Col. San Pedro Zacatenco, A.P. 17740, 07300 México, D.F., Mexico

hsira@cinvestav.mx

ABSTRACT. An algebraic framework for continuous-time linear systems identification introduced in the literature some years ago has revealed as an interesting alternative way for on-line parametric identification. The present contribution aims at conveying those ideas to linear timeinvariant discrete-time systems, with particular emphasis attached to application issues. To this end, an on-line linear identifier for $n$-th order systems is evolved, re-sorting to the operational representation of the dynamics. Being discussed on the basis of a fifth-order model of a drive-train, the numerical condition of the obtained setting of the identifier is found to suffer significantly with decreasing sampling times. A setting not experiencing these numerical problems is finally introduced by means of a re-parametrization of the identifier via application of

RS - JESA - 42/2008. Identification des systèmes, pages 210 à 232 
the bilinear Tustin transform. The already implemented computer programs, where computer algebra plays an important role, are available.

RÉSUMÉ. Cette communication est le pendant discret d'une technique algébrique récente d'identification paramétrique pour systèmes linéaires stationnaires et continus. On y accorde une importance particulière aux applications, en calculant, grâce à la transformation en z, un identifieur en ligne pour un système d'ordre n. Le dispositif de laboratoire est d'ordre cinq. La transformation bilinéaire de Tustin permet de contrecarrer l'instabilité numérique due à un échantillonnage trop rapide. Les programmes de mise en æuvre, où le calcul formel joue un rôle important, sont disponibles.

KEYWORDS: Discrete-time linear systems, z-transform, bilinear Tustin transform, parameter identification, on-line identifier, rapid sampling, computer algebra.

MOTS-CLÉS : Systèmes linéaires discrets, transformation en z, transformation bilinéaire de Tustin, identification paramétrique, identifieur en ligne, échantillonnage rapide, calcul formel. 


\section{Introduction}

This article, the origin of which is a recent conference communication (Fliess et al., 2006), further develops recent works on discrete-time parametric identification (see (Sira-Ramírez et al., 2002; Fliess et al., 2002; Fuchshumer, 2006). It is a counterpart, augmented with application issues, of (Fliess et al., 2003; Fliess et al., 2008b), which permits for linear time-invariant continuous-time systems, thanks to algebraic methods, to achieve,

- on-line parametric identification,

- robustness with respect to noisy data without knowing the statistical properties of the corrupting noises (see (Fliess, 2006) for further details).

In accordance to the continuous-time framework, the operational representation of the discrete-time constant linear system (in the $z$-domain) is considered. Initial conditions are allowed to being ignored by taking derivatives with respect to the shift operator $z$. To determine the unknown system parameters, subsequent iterated summations of the discrete-time counterpart of the resulting operational equation are carried out to set up a system of linear equations, referred to as linear identifier. The presentation is evolved for a general $n$-th order discrete-time constant linear dynamics. To cope with measurement noise, besides the possibility of a straightforward incorporation of linear filters, the setup of an over-determined system of linear equations by means of additional iterated summations qualifies to be suitable.

On the basis of a fifth-order model of a drive-train, which is available as a laboratory experiment, the problem of inaccurate estimation of those system zeros, which have only minor effect on the system response, and, hence, are difficult to estimate in presence of noise, is illustrated. Additionally, it is found that the identifier parameterized in terms of the $z$-domain parameters, i.e., the coefficients of the system's difference equation, exhibits increasingly poor numerical condition emerging with decreasing sampling times. This numerical issue of the $z$-domain setting might become apparent by reflecting the relation $z_{i}=\exp \left(s_{i} T_{a}\right)$ between the poles $s_{i}$ of the continuous-time system and the poles $z_{i}$ of the according discrete-time representation. Hence, with decreasing sampling time $T_{a}$, the poles $z_{i}$ approach the point $z=1$. In order to overcome this numerical problem, a suitable re-parametrization of the identifier is sought for and found in terms of the bilinear Tustin transform, also referred to as $q$-transform for short in linear systems theory (see, e.g., (Middleton et al., 1990) for a related transform). Again referring to the drive-train example it is shown that the $q$-domain setting of the identifier does not experience numerical deficiencies in case of small sampling times.

To cope with the problem of inaccurate estimation of "inessential" zeros, the idea to discarding (or pre-setting) those zeros, based on a priori knowledge from modeling, is proposed. This approach is first discussed on the basis of the $q$-domain setting and then transferred to the $z$-domain framework accordingly. The motivation for discussing this idea for the $q$-domain case first simply is that, by virtue of the close sim- 
ilarity to the continuous-time domain, things might be particularly apparent from the control engineer's point of view. This idea of pre-setting those non-essential zeros to obtain a "reduced-order" linear identifier is shown to provide particular attractiveness. The corresponding computer programs are available.

The accuracy of our identification results is demonstrated by inspecting the tight correspondence of measurement and simulation of a drive-train control application, with the controller designed on the basis of the identified dynamics using a simple loop-shaping procedure (see, e.g., (Gausch et al., 1991; Longchamp, 2006)).

\section{A linear identifier for $n$-th order discrete-time SISO LTI systems}

Consider a linear time-invariant discrete-time SISO system of order $n$

$$
\sum_{i=0}^{n} a_{i} y_{k+i}=\sum_{i=0}^{m} b_{i} u_{k+i}, \quad m \leq n, \quad a_{n}=1
$$

For estimating the unknown parameters

$$
\lambda^{T}=\left[a_{0}, \ldots, a_{n-1}, b_{0}, \ldots, b_{m}\right]
$$

we start with the $z$-domain representation of system [1], which yields an identification approach motivated by (Fliess et al., 2003; Fliess et al., 2008b).

Consider a sequence $\left(f_{k}\right)$, where $f_{k}=0$ for $k<0$. The formal Laurent series $f_{z}(z)=\sum_{i=0}^{\infty} f_{i} z^{-i}$, called the $z$-transform of $\left(f_{k}\right)$, is written $f_{z} \bullet \bullet\left(f_{k}\right)$ for short. With $\left(f_{k+i}\right) \circ \bullet z^{i}\left(f_{z}-\sum_{j=0}^{i-1} f_{j} z^{-j}\right)$, and $A(z)=z^{n}+\sum_{i=0}^{n-1} a_{i} z^{i}, B(z)=$ $\sum_{i=0}^{m} b_{i} z^{i}$, the $z$-domain counterpart of Equation [1] is

$$
A(z) y_{z}-\sum_{i=0}^{n} a_{i} z^{i} \sum_{j=0}^{i-1} y_{j} z^{-j}=B(z) u_{z}-\sum_{i=0}^{m} b_{i} z^{i} \sum_{j=0}^{i-1} u_{j} z^{-j}
$$

and the associated transfer function is

$$
G(z)=\frac{y_{z}(z)}{u_{z}(z)}=\frac{B(z)}{A(z)}
$$

Correspondingly to the continuous-time framework of (Fliess et al., 2003; Fliess et al., 2008b), derivatives with respect to $z$, of order $\bar{n} \geq n+1$, are taken on both sides of Equation [3] in order to eliminate the initial conditions $y_{j}, j=0, \ldots n-1$, and $u_{j}, j=0, \ldots m-1$. (Accordingly, one might think, e.g., of first dividing both sides of Equation [3] by $z$, followed by an $n$ times differentiation with respect to $z$, in order to meet this objective.) 
By carrying out the derivative of order $\bar{n} \geq n+1$ on both sides of Equation [3] (the detailed calculations are given in the appendix) and finally transferring the result back to the discrete-time domain, we end up with

$$
\left(\prod_{s=0}^{\bar{n}-1}(k-n+s)\right)\left\{y_{k}+\sum_{i=0}^{n-1} y_{k-n+i} a_{i}-\sum_{i=0}^{m} u_{k-n+i} b_{i}\right\}=0
$$

For determining the set $\lambda$ of parameters, Equation [5] is subject to $N \geq n+m$ fold iterated summation (correspondingly to the iterated integrals of (Fliess et al., 2003)) to assemble a set of $(N+1)$ linear equations, $P(k) \lambda=Q(k)$, also referred to as an (on-line) linear identifier. So, with $\chi(k)=\prod_{s=0}^{\bar{n}-1}(k-n+s)$, the first row of $P$ and $Q$ is given as $P_{1, i+1}(k)=\chi(k) y_{k-n+i}, i=0, \ldots, n-1, P_{1, n+j+1}(k)=$ $-\chi(k) u_{k-n+j}, j=0, \ldots, m, Q_{1}(k)=-\chi(k) y_{k}$. The subsequent rows read $P_{1+l, i}(k)=\sum_{r=0}^{k-1} P_{l, i}(r), l=1, \ldots, N, i=1, \ldots, n+m+1$, with an according expression for $Q$. The reason for optionally setting up an over-determined system of linear equations (by choosing $N>n+m$ ) is the following: it provides an improved accuracy for the estimates in presence of noisy signals. Section 5 will deal with some related issues.

\section{Re-parameterizing the problem via application of the Tustin transform}

Given a continuous-time system $G(s)$ with poles $s_{i}$, then the poles of the according discrete-time system $G(z)$ are located at $z_{i}=\exp \left(s_{i} T_{a}\right)$. Thus, with a decreasing sampling time $T_{a}$, the poles $z_{i}$ approach 1 . The objective of the following discussions is to introduce a suitable re-parametrization of the proposed identification method, namely via application of the bilinear Tustin transform $\mathbb{C} \rightarrow \mathbb{C}$,

$$
z=\frac{1+q / \Omega_{0}}{1-q / \Omega_{0}}, \quad q=\Omega_{0} \frac{z-1}{z+1}, \quad \Omega_{0}=\frac{2}{T_{a}}
$$

in order to avoid poor numerical conditioning emerging with decreasing sampling times. Section 6.1 will illustrate these (numerical) issues on the basis of a "drivetrain" example.

\section{Let}

$$
\dot{G}^{\#}(q)=\left.G(z)\right|_{z=\frac{1+q / \Omega_{0}}{1-q / \Omega_{0}}}
$$

denote the $q$-domain transfer function, with the prime arranged to indicate the representation in terms of $\lambda$. Clearly, an $(n-m)$-fold zero at $q=\Omega_{0}$ occurs. Next, arrange a change of the parameters to re-cast $G^{\#}(q)$ as

$$
G^{\#}(q)=\frac{\left(1-q / \Omega_{0}\right)^{n-m} \sum_{i=0}^{m} B_{i} q^{i}}{1+\sum_{i=1}^{n} A_{i} q^{i}}
$$


represented in terms of the parameters $\left\{A_{i}, B_{i}\right\}$. Notice that, given a continuous-time system $G(s)$, the approximation $G^{\#}(j \Omega) \approx G(j \omega)$, with $\Omega=\left(2 / T_{a}\right) \tan \left(\omega T_{a} / 2\right)$, holds for $\left|\omega T_{a}\right| \ll 1$. Let $s=a$ be a pole of $G(s)$, then the according pole of $G^{\#}(q)$ is $\tilde{a}=\Omega_{0} \tanh \left(a / \Omega_{0}\right)$. This observation draws a close link between the $q$-domain and the $s$-domain scenario.

The following idea will be crucial for additionally increasing the performance of the identifier (to be illustrated in Section 6.1). Being particularly apparent in the $q$ domain setting from the control engineer's point of view, one may discard, except for the $(n-m)$-fold zero at $\Omega_{0}$, those zeros of $G^{\#}(q)$ which only have minor influence on the system response, and, hence, are difficult to estimate in presence of noise. This idea of discarding the "inessential" zeros a priori (based on knowledge obtained from modeling) will be accordingly transferred to the $z$-domain setting in Section 4.

So, the point of departure for the $q$-domain setting of the identification approach, incorporating the (optional) feature of discarding "inessential" zeros, is set as

$$
\tilde{G}^{\#}(q)=\frac{\left(1-q / \Omega_{0}\right)^{n-m} \sum_{i=0}^{\tilde{m}} \tilde{B}_{i} q^{i}}{1+\sum_{i=1}^{n} A_{i} q^{i}}, \quad \tilde{m} \leq m
$$

with the tilde indicating the approximation of $G^{\#}(q)$. Next, we will trace back to the $z$-domain solution, and re-cast [5] in terms of the $q$-domain parameters

$$
\Lambda^{T}=\left[A_{1}, \ldots, A_{n}, \tilde{B}_{0}, \ldots, \tilde{B}_{\tilde{m}}\right]
$$

To this end, let us first determine the parameter transformation. The $z$-domain transfer function (with $a_{n} \neq 1$ in general) according to $\tilde{G}^{\#}(q)$ reads

$$
G(z)=\frac{2^{n-m} \sum_{i=0}^{\tilde{m}} \tilde{B}_{i} \Omega_{0}^{i}(z-1)^{i}(z+1)^{m-i}}{(z+1)^{n}+\sum_{i=1}^{n} A_{i} \Omega_{0}^{i}(z-1)^{i}(z+1)^{n-i}}=\frac{\sum_{s=0}^{m} b_{s} z^{s}}{\sum_{s=0}^{n} a_{s} z^{s}}
$$

By referring to the binomial theorem, we obtain the relations between the $(n+m+2)$ coefficients $\left\{a_{s}, b_{s}\right\}$ of [11] and the $(n+\tilde{m}+1)$ parameters $\Lambda$ as

$$
\begin{aligned}
& b_{s}=\quad \sum_{i=0}^{\tilde{m}} \Pi_{s, i}^{b} \tilde{B}_{i}, \quad s=0, \ldots, m \\
& a_{s}=\left(\begin{array}{c}
n \\
s
\end{array}\right)+\sum_{i=1}^{n} \Pi_{s, i}^{a} A_{i}, \quad s=0, \ldots, n
\end{aligned}
$$

with

$$
\begin{array}{ll}
\Pi_{s, i}^{b}=2^{n-m}\left(-\Omega_{0}\right)^{i} \sum_{j=0}^{s}(-1)^{s+j}\left(\begin{array}{c}
i \\
s-j
\end{array}\right)\left(\begin{array}{c}
m-i \\
j
\end{array}\right), \\
\Pi_{s, i}^{a}=\quad\left(-\Omega_{0}\right)^{i} \sum_{j=0}^{s}(-1)^{s+j}\left(\begin{array}{c}
i \\
s-j
\end{array}\right)\left(\begin{array}{c}
n-i \\
j
\end{array}\right)
\end{array}
$$


Plugging the transformation [12,13] into [5] (notice that [5] has to be slightly adapted so as to allow for arbitrary $a_{n}$ ), we finally find

$$
\begin{aligned}
\chi(k)\left\{\sum_{r=0}^{n}\left(\begin{array}{l}
n \\
r
\end{array}\right) y_{k-n+r}+\sum_{i=1}^{n}\left(\sum_{r=0}^{n} y_{k-n+r} \Pi_{r, i}^{a}\right) A_{i}-\right. & \\
& \left.-\sum_{i=0}^{\tilde{m}}\left(\sum_{r=0}^{m} u_{k-n+r} \Pi_{r, i}^{b}\right) \tilde{B}_{i}\right\}=0
\end{aligned}
$$

The procedure of setting up the linear identifier by means of iterated summation of [14], and the solution for $\Lambda$ as well, proceeds as discussed in Section 2.

\section{The $z$-domain setting - cont'd}

The idea to (optionally) a priori discarding "inessential" zeros will now be equivalently applied to the $z$-domain setting, i.e., the parametrization in terms of $\lambda$, continuing Section 2. Clearly, by [6], discarding certain zeros of $G^{\#}(q)$, i.e., shifting those zeros to infinity, corresponds to placing the associated zeros of $G(z)$ at $z=-1$. Now, introduce the counterpart of [9],

$$
\tilde{G}(z)=\frac{(z+1)^{m-\tilde{m}} \sum_{i=0}^{\tilde{m}} \tilde{b}_{i} z^{i}}{\sum_{i=0}^{n} a_{i} z^{i}}=\frac{\sum_{i=0}^{m} b_{i} z^{i}}{\sum_{i=0}^{n} a_{i} z^{i}}
$$

with the tilde indicating the approximation of $G(z)$. The linear map relating the parameters $\tilde{b}^{T}=\left[\tilde{b}_{0} \ldots \tilde{b}_{\tilde{m}}\right]_{\tilde{\lambda}^{T}}$ to the numerator coefficients $b^{T}=\left[b_{0} \ldots b_{m}\right]$ is represented as $b=\Xi_{b} \tilde{b}$. Let $\tilde{\lambda}^{T}=\left[a_{0}, \ldots, a_{n-1}, \tilde{b}_{0}, \ldots, \tilde{b}_{\tilde{m}}\right]$, then $\lambda=\Xi \tilde{\lambda}$ with $\Xi=\operatorname{diag}\left(E^{n \times n}, \Xi_{b}\right)$, and the linear identifier reads $\tilde{P} \tilde{\lambda}=Q$, with $\tilde{P}=P \Xi$.

\section{Robustness with respect to noisy data}

In presence of noisy signals, the composition of the identifier as a "square" system of linear equations, i.e., $N=n+m$ or $N=n+\tilde{m}$ respectively, via iterated summation of [5] or [14], might not yield accurate (or even appropriate) estimates. For instance, this is the case for the drive-train example to be investigated in Section 6.1. We will address here some possibilities (which might of course be combined) for improving the performance of the proposed identification method in presence of noisy signals:

1) instead of taking iterated summations on [5] or [14], i.e., iterated application of $1 /(z-1)$ in the $z$-domain, one might more generally iteratively apply (filters of the type) $1 /(z-\gamma)$ (or even higher-order ones) for denoising,

2) "invariant filtering" (see, e.g., (Fliess et al., 2008b)): Each row of the linear identifier $P(k) \lambda=Q(k)$ (or its counterparts of Sections 3 and 4) might be preprocessed with a filter, suitably adjusted utilizing (a priori) knowledge of the system dynamics, 
3) instead of setting up a "square" linear identifer, one might choose $N \gg n+m$ (or $N \gg n+\tilde{m}$, resp.) to set up an over-determined system (in the presence of noise) and solve, e.g., for a least-squares solution to (the under-determined linear system) $P \lambda-e=Q$, i.e., $\min _{\lambda}\|e\|_{2}^{2}$, which is $\lambda=\left(P^{T} P\right)^{-1} P^{T} Q$,

4) clearly, the "first few" equations of the linear identifier are obviously more affected by noises than the subsequent ones. This is due to the effect of the iterated summations (or the iterated filters of item 1). So, one might think of discarding the "first" $\alpha$ equations from the parameter calculation.

The combination of the items 3 and 4 might read as follows: Set up a number of $N=n+\tilde{m}+\alpha+\beta$ equations (either by means of iterated summation or filtering), with $\beta$ denoting the number of additional equations added due to item 3 . Hence, from the $(1+N)$ equations, the equations no. $(\alpha+1)$ up to $(1+N)$, i.e., a number of $1+N-\alpha=1+n+\tilde{m}+\beta$ equations, are used for calculating the $(1+n+\tilde{m})$ unknown parameters (in the least-squares sense).

REMARK. - Several aspects of the above discussion are nothing else but a discretetime interpretation of the viewpoint expounded in (Fliess, 2006), where noises are considered as highly fluctuating, or oscillating, phenomena. Let us emphasize once more that we do not need any statistical knowledge of the noises.

REMARK. - See (Mboup, 2006; Mboup, 2007) for a most illuminating comparison between continuous-time algebraic identification methods and least-square techniques.

\section{Application and discussion}

\subsection{Identification}

To illustrate the behavior of the presented approach to discrete-time linear systems identification, and, in particular, to reveal some subtleties involved, we will finally discuss a selected application available as a laboratory experiment.

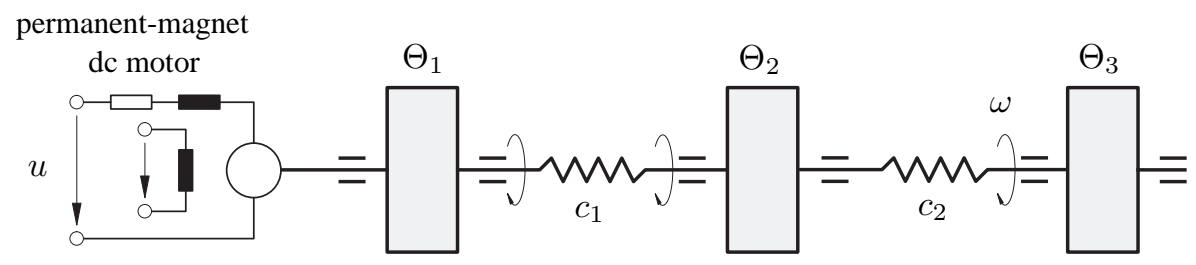

Figure 1. The lab model "drive-train"

Consider the model of a drive train as depicted in Figure 1. The parameters of the lab setup are as follows: $L_{A}=896 \mu H$ (armature inductance), $R_{A}=6.38 \Omega$ 
(armature resistance), $k_{m}=41 \cdot 10^{-3} \mathrm{Nm} / A$ (torque constant), $c_{1}=c_{2}=$ $1.72 \cdot 10^{-3} \mathrm{Nm} / \mathrm{rad}$ (spring coefficients), $\Theta_{1}=25.65 \cdot 10^{-6} \mathrm{kgm}^{2}, \Theta_{2}=$ $6.44 \cdot 10^{-6} \mathrm{kgm}^{2}, \Theta_{3}=5.1 \cdot 10^{-6} \mathrm{kgm}^{2}$ (moments of inertia of the rotors), and $d_{1}=3.98 \cdot 10^{-6} \mathrm{Nms}, d_{2}=0.92 \cdot 10^{-6} \mathrm{Nms}, d_{3}=2.4 \cdot 10^{-6} \mathrm{Nms}$ (coefficients of viscous friction, related to the bearings of the respective rotors). By discarding the dynamics related to the electrical subsystem in the sense of a singular perturbation point of view, the dynamics of the drive-train are obtained as $G(s)=\hat{\omega} / \hat{u}$,

$$
G(s)=\frac{V}{\left(1+\frac{s}{8.1}\right)\left(1+\frac{2 \xi_{1} s}{\omega_{1}}+\frac{s^{2}}{\omega_{1}^{2}}\right)\left(1+\frac{2 \xi_{2} s}{\omega_{2}}+\frac{s^{2}}{\omega_{2}^{2}}\right)}
$$

with $V=23.7, \omega_{1}=12.4, \omega_{2}=27.7, \xi_{1}=0.1$ and $\xi_{2}=0.0083$. Thus $n=5$, $m=4$ for the according discrete-time system $G(z)$.

The following examples (with $\bar{n}=n+1$ ) provide different case studies regarding the choice of the parameter $\tilde{m} \leq m$ (for both the $q$-and $z$-domain setting) and the sampling time $T_{a}$. In order to cope with noise, the combination of the items 3 and 4 of Section 5 is applied, with $\alpha=7$ and $\beta=10$. Numerous case studies showed that for this example, the use of the iterated summations (i.e., $\gamma=1$ ) is clearly advantageous compared to the choice $\gamma \neq 1$ of item 1 (of Section 5). The "invariant filtering approach" of item 2 was found not to give significant further improvements (to the setting as introduced above), so it is not applied within the following case studies.

All equations of the identifier are normalized (by dividing by the maximum absolute entry of $P$ of the respective row) to improve the numerical conditioning. The linear on-line identifiers start at $t=0$, and the pole-zero plots and Bode diagrams of the identified $z$ - and $q$-domain transfer functions given in the figures are due to $\lambda$ and $\Lambda$, evaluated at the final time $t_{\text {end }}=1.35 \mathrm{~s}$. The pole-zero plots of the nominal dynamics $G(z)$, or $G^{\#}(q)$ resp., associated to $G(s)$ as given above, will always be displayed in blue color.

Example 1 Set $\tilde{m}=m, T_{a}=10 \mathrm{~ms}$. The simulation results given in the Figures 2 and 3 are associated with the observation that, in presence of noise (chosen as colored), the estimation of the zeros which have minor influence on the system response (see the subplot containing the system output $\omega$ ) is very poor. Additionally, it is found that, emerging with decreasing sampling times, the numerical conditioning of the linear identifier parameterized in terms of the $z$-domain parameters $\lambda$ becomes increasingly worse, in contrast to the q-domain setting. To illustrate this, the conditioning numbers of $P$ (i.e., the ratio of the largest singular value of $P$ to the smallest) for the $z$-and q-domain identifier, evaluated at the final time $t_{\text {end }}$, for different sampling times, are given in Table 1 (cols. 2 and 3 ).

In order to show that these conditioning numbers are only weakly affected by the noise added to the output signal, the conditioning numbers obtained by noise-free simulations are also given in Table 1 (cols. 4 and 5). 


\begin{tabular}{c|cc|cc}
$T_{a}[\mathrm{~ms}]$ & $z$ & $q$ & $z$ & $q$ \\
\hline 10 & $3.0 e 10$ & $1.7 e 10$ & $3.0 e 10$ & $1.7 e 10$ \\
5 & $5.1 e 11$ & $8.0 e 9$ & $5.2 e 11$ & $7.8 e 9$ \\
2 & $2.9 e 13$ & $1.2 e 10$ & $2.9 e 13$ & $1.2 e 10$ \\
1 & $5.4 e 14$ & $1.4 e 10$ & $5.4 e 14$ & $1.4 e 10$ \\
0.5 & $9.0 e 15$ & $1.5 e 10$ & $8.9 e 15$ & $1.5 e 10$
\end{tabular}

Table 1. (see Example 1) Conditioning numbers of $P$

The first observation of Example 1 (inappropriate estimation of the zeros in presence of noise), associated with the knowledge obtained from modeling, is the motivation for applying the approximations of the numerators as proposed in Sections 3, 4. Notice that, by inspecting for instance the Bode plots of the nominal drive-train dynamics in Figure 3, those mentioned zeros only affect the frequency response in the frequency domain with the magnitude $\left|G^{\#}(j \Omega)\right|$ located significantly below the zero $\mathrm{dB}$ line. The idea of discarding those zeros is addressed in the following example.

Example 2 Set $\tilde{m}=0$ and $T_{a}=10 \mathrm{~ms}$ for the simulation results given in the Figures 4 and 5. The approach for discarding those zeros having negligible effect on the system response is seen to be appropriate (see in particular the subplot containing the system output $\omega$ ). The estimation of the poles is again accurate. Additionally, due to the reduced number of parameters, this approach has also the advantage of having better numerical conditioning compared to the case $\tilde{m}=m$ of the previous example, illustrated by the condition numbers given in Table 2. Analogously to Table 1, cols. 4

\begin{tabular}{c|cc|cc}
$T_{a}[\mathrm{~ms}]$ & $z$ & $q$ & $z$ & $q$ \\
\hline 10 & $1.8 e 7$ & $2.3 e 7$ & $1.9 e 7$ & $2.3 e 7$ \\
5 & $6.0 e 8$ & $2.7 e 7$ & $5.8 e 8$ & $2.7 e 7$ \\
2 & $3.5 e 10$ & $3.0 e 7$ & $3.5 e 10$ & $3.0 e 7$ \\
1 & $6.4 e 11$ & $3.1 e 7$ & $6.4 e 11$ & $3.1 e 7$ \\
0.5 & $1.1 e 13$ & $3.2 e 7$ & $1.1 e 13$ & $3.2 e 7$
\end{tabular}

Table 2. (see Example 2) Conditioning numbers of $P$

and 5 display the results obtained with noise-free simulations. Again, the numerical conditioning of the $z$-domain setting suffers with decreasing sampling times, whereas the parametrization in terms of $\Lambda$ does not show such problems.

Example 3 (measurement results) Set $\tilde{m}=0, T_{a}=10 \mathrm{~ms}$. Figures 6 and 7 depict the identification results obtained from measurements of the "drive-train" lab model. The angular velocity $\omega$ is measured via an incremental encoder. 

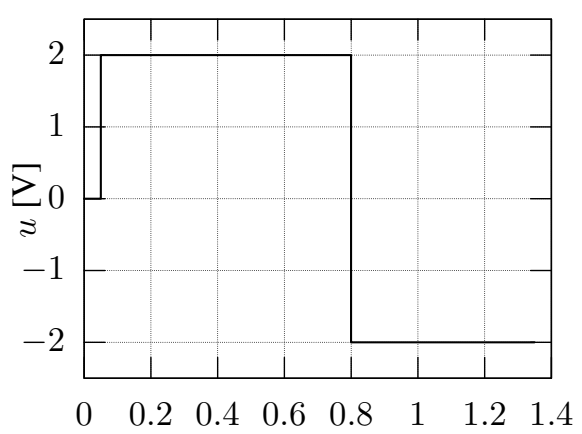

$t[\mathrm{~s}]$
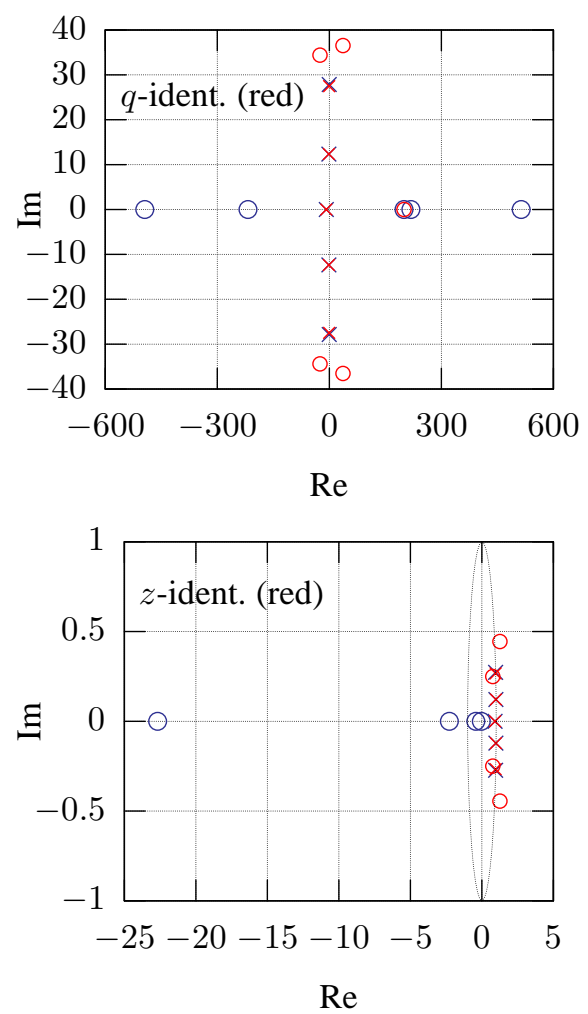
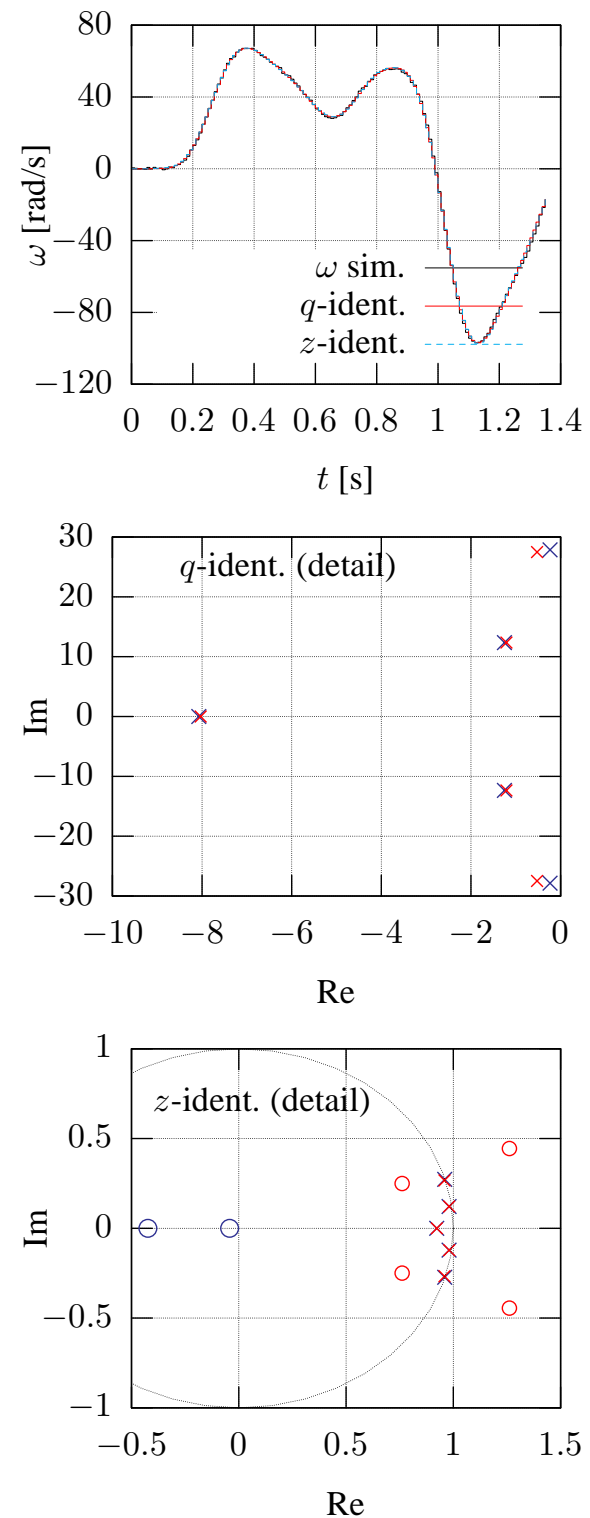

Figure 2. (see Example 1) Simulation results, $\tilde{m}=m, T_{a}=10 \mathrm{~ms}$. Both $z$-and $q$-domain setting provide good results (regarding the system dynamics in the "interesting" frequency domain, see the system response and the Bode diagrams of Figure 3. However, the estimation of the zeros of both parameterizations is very inappropriate indeed 

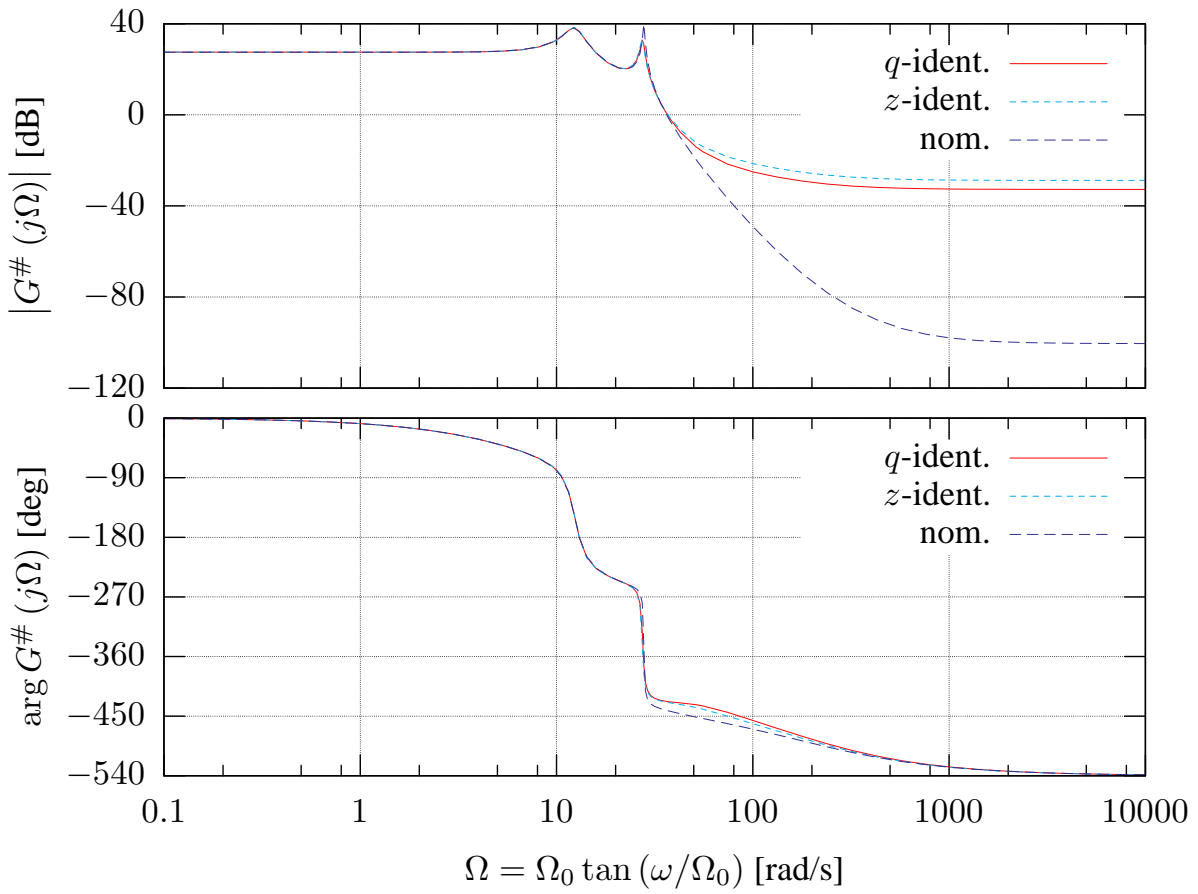

Figure 3. (see Example 1) Figure 2 cont'd: Bode plots

Let us end these case studies with some considerations on the counterparts of the Examples 1 and 2 by invoking a standard least-squares (LS) method in the $z$ - and the $q$-domain. We also present a comparison with an instrumental-variable (IV4) identification method (see, e.g., (Ljung, 1999; Söderström et al., 1989)) in the $z$-domain, since by assumption the equation error is not white noise.

Example 4 (standard least-squares $(L S)$ method; $\tilde{m}=m, T_{a}=10 \mathrm{~ms}$ ). The results obtained with the same measurement data as given in Figure 6 are displayed in Figure 8. They indicate poor performance (getting worse with decreasing sampling times), even for the estimation of the pole-pair related to the "slow eigenfrequency".

Example 5 (standard LS method; $\tilde{m}=0, T_{a}=10 \mathrm{~ms}$ ). This setting is found not to give any clear improvements compared to the choice $\tilde{m}=m$ of Example 4, thus, the associated graphics are not displayed.

REMARK. - In order to achieve suitable results with the LS identification (in the case of the drive-train example), it is advisable to increase the sampling time, say, e.g., to $T_{a}=50 \mathrm{~ms}$, additionally to articulately increasing the "observation time span". 

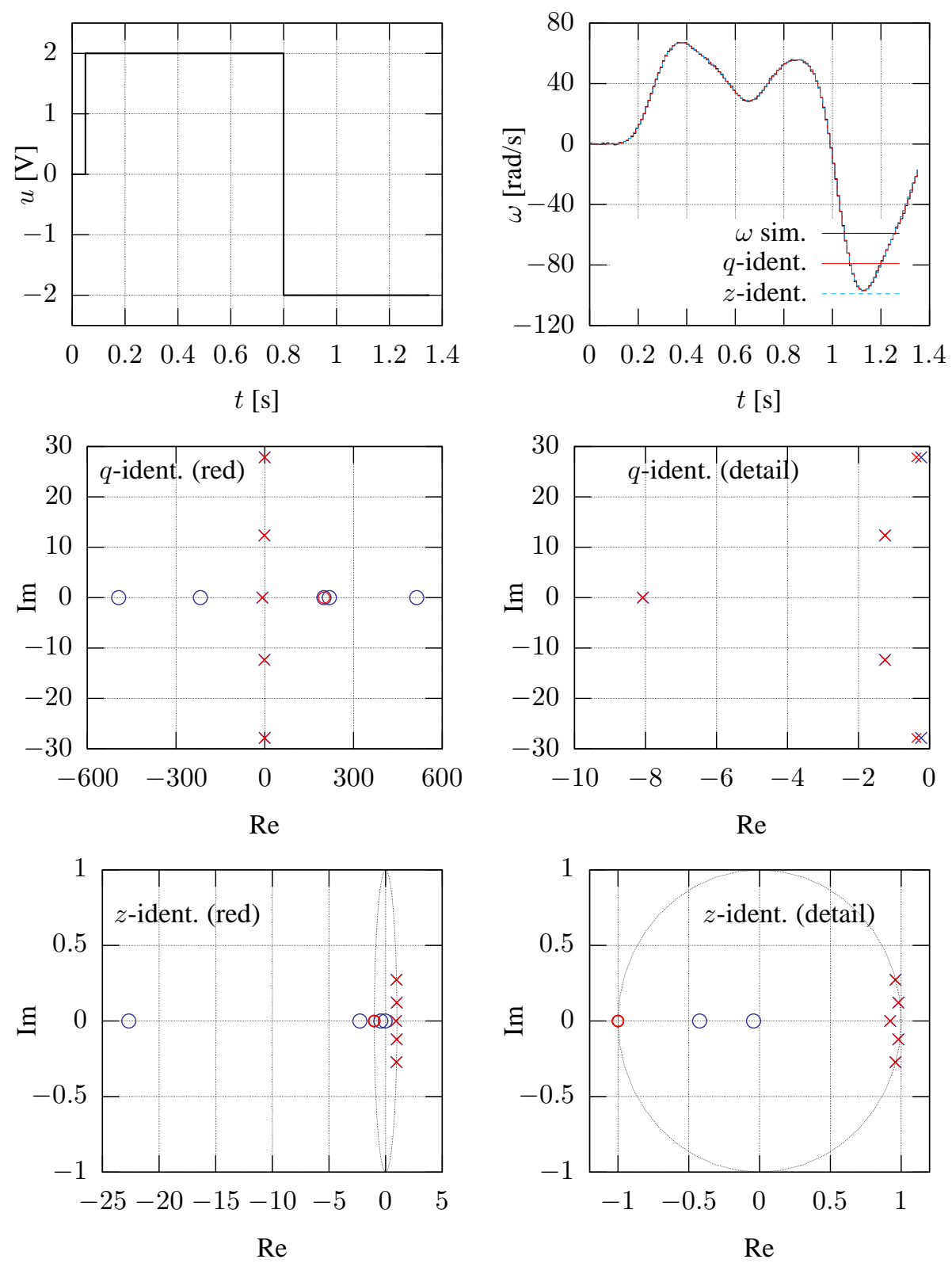

Figure 4. (see Example 2) Simulation results, $\tilde{m}=0, T_{a}=10 \mathrm{~ms}$. The usefulness of the idea of approximating the numerator in the sense of Sections 3 and 4 is verified, see also Figure 5 for the Bode plots 

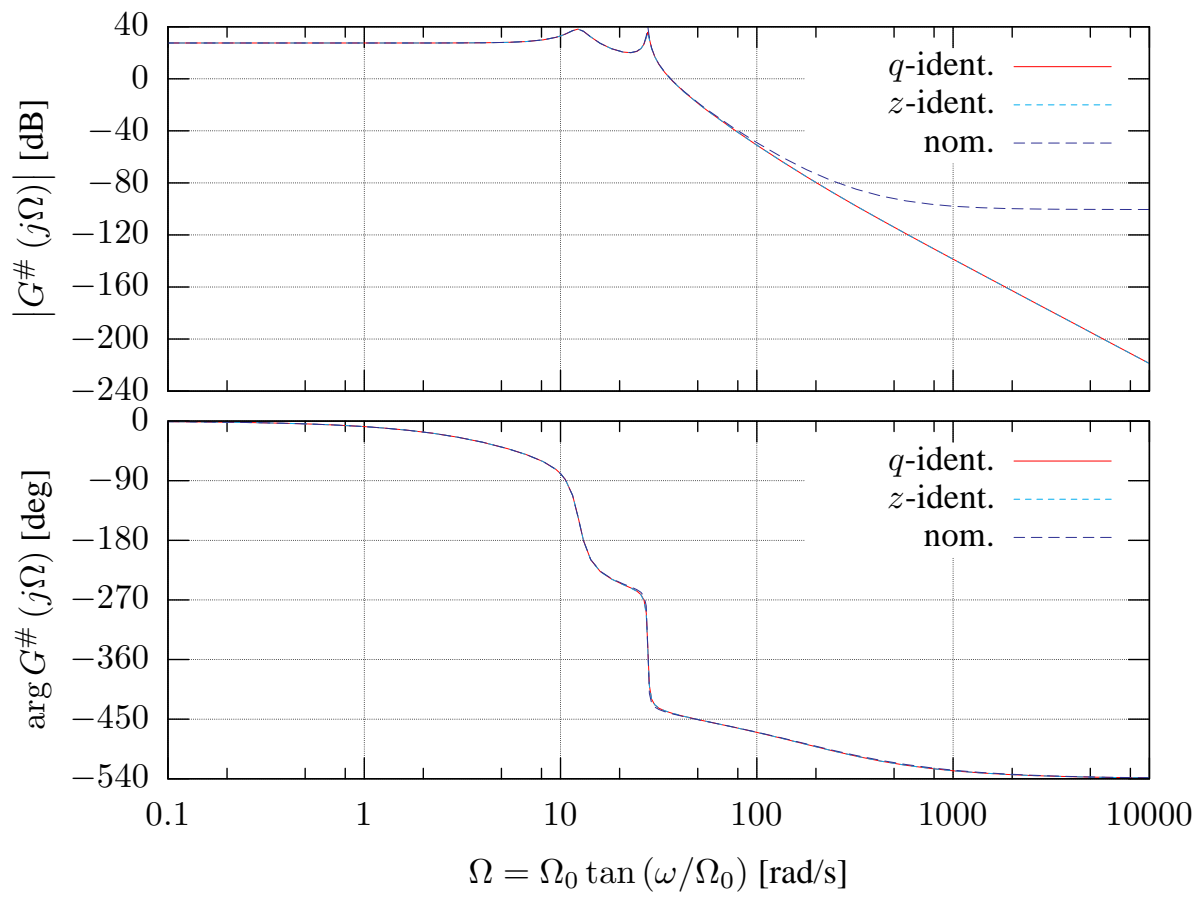

Figure 5. (see Example 2) Figure 4 cont'd: Bode plots

Example 6 (IV4 method $\tilde{m}=m, T_{a}=10 \mathrm{~ms}$ ). The results obtained with the same measurement data as given in Figure 6 are displayed in Figure 9.

REMARK. - The use of colored noises is a further confirmation that our denoising techniques are not limited to classic Gaussian white noises ${ }^{1}$.

\subsection{Control application}

Based on the identification result of the drive-train lab model displayed in the Figures 6 and 7, a controller $R$ (for the one-degree-of-freedom standard control scheme) is now designed by means of a loop-shaping procedure, i.e., a simple graphical method based on Bode plots (see, e.g., (Gausch et al., 1991; Longchamp, 2006)). The design is carried out in the $q$-domain. To this end, the transfer function (i.e., the amplitude

1. See (Fliess, 2006) and the references therein for confirmation in various areas including signal processing (see also (Neves et al., 2007; Trapero et al., 2007; Trapero et al., 2008)). 

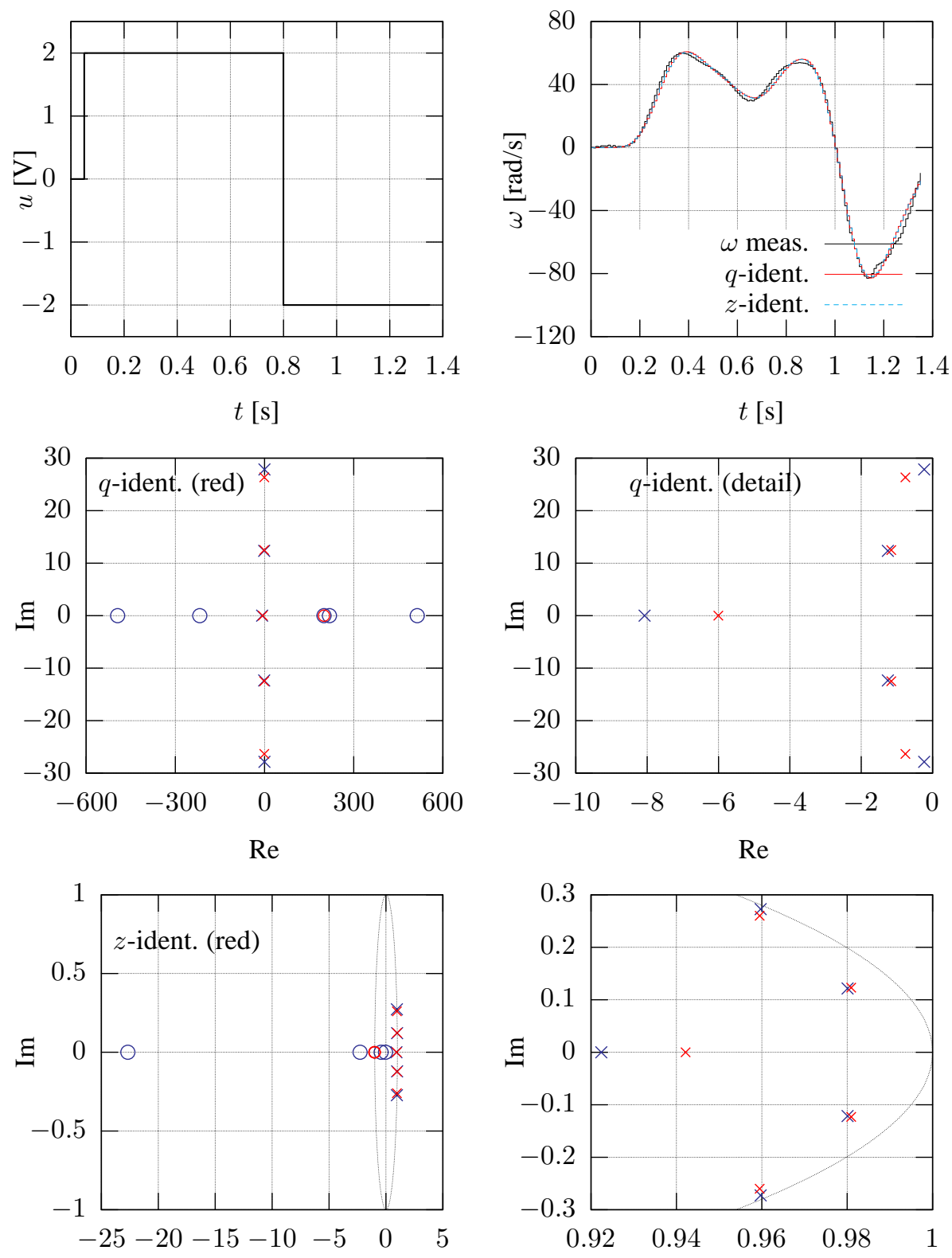

$\operatorname{Re}$

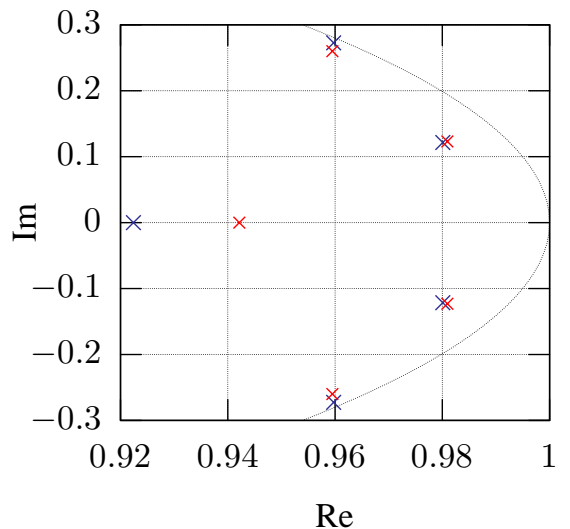

Figure 6. (see Example 3) Measurement results, $\tilde{m}=0, T_{a}=10 \mathrm{~ms}$. See also Figure 7 for the Bode plots 

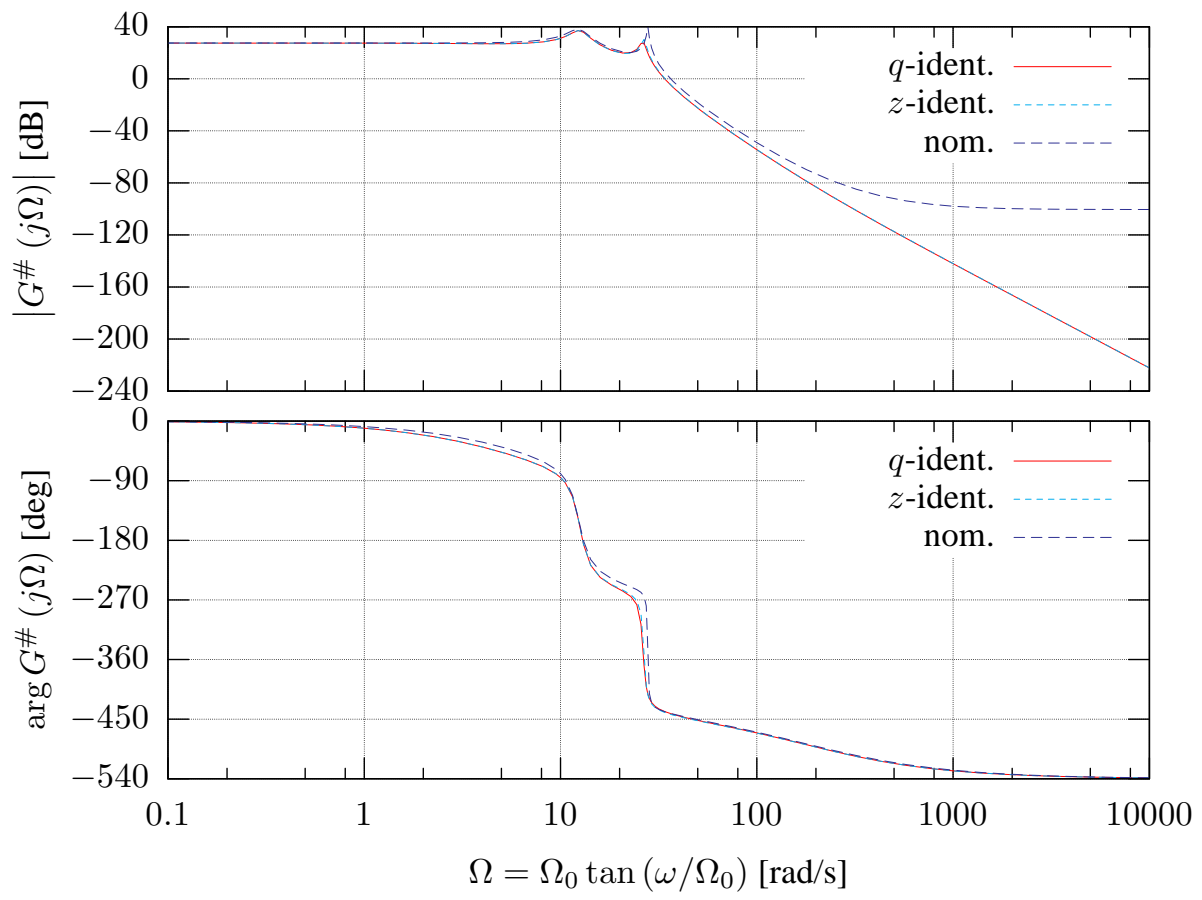

Figure 7. (see Example 3) Figure 6 cont'd: Bode plots

and phase response) of $L=R G$ of the open loop is given a suitable shape so as to meet the closed loop design objectives.

The following control design may be retraced via the Bode plots of Figure 10. Let $G^{\#}(q)$ denote the identified $q$-domain transfer function of the drive-train due to Figure 7. As the first design step, in order to cope with the fairly-damped torsional oscillations of the drive-train system, the according complex-conjugate polepairs $-1.16 \pm 12.5 \sqrt{-1}$ and $-0.76 \pm 26.34 \sqrt{-1}$ of $G^{\#}(q)$ are compensated for. Let us represent the polynomials associated with those pole-pairs as $1+2 \xi_{i} q / \omega_{i}+\left(q / \omega_{i}\right)^{2}$, $i=1,2$, with $\left(\omega_{1}=12.55 \mathrm{rad} / \mathrm{s}, \xi_{1}=0.0923\right)$ for the "slow" eigenfrequency and $\left(\omega_{2}=26.35 \mathrm{rad} / \mathrm{s}, \xi_{2}=0.0289\right)$ for the "fast" eigenmode, respectively. Typically, in view of robustness issues regarding performance, it is advisable not to exactly cancel out such pole-pairs $1+2 \xi_{i} q / \omega_{i}+\left(q / \omega_{i}\right)^{2}$, but to install (approximate) compensators, as, e.g.,

$$
N_{i}^{\#}(q)=\frac{1+2 \bar{\xi}_{i} q / \omega_{i}+\left(q / \omega_{i}\right)^{2}}{1+2 \zeta_{i} q / \omega_{i}+\left(q / \omega_{i}\right)^{2}}
$$




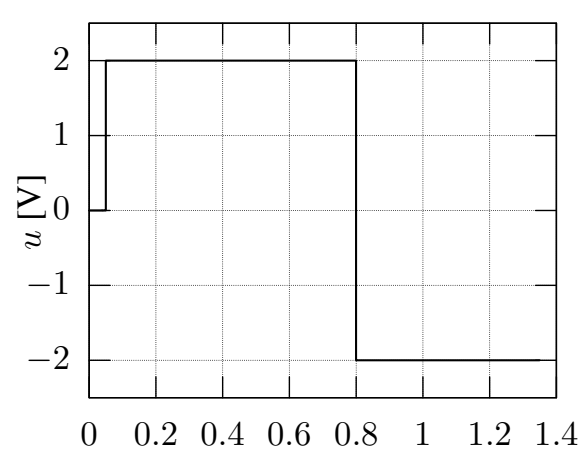

$t[\mathrm{~s}]$

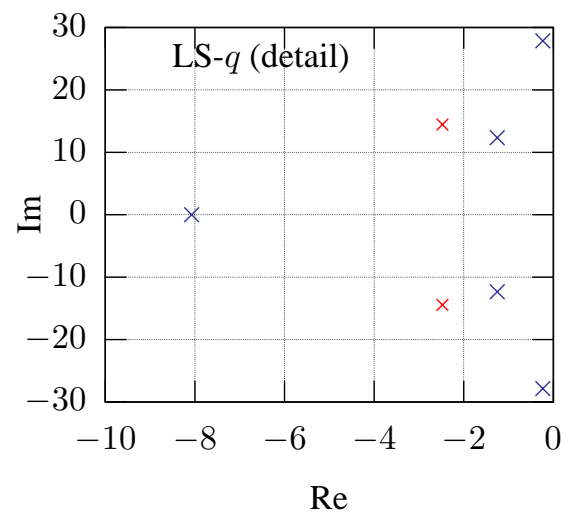

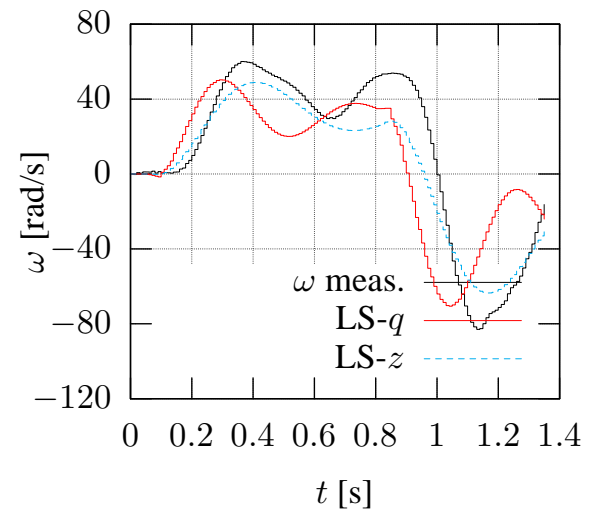

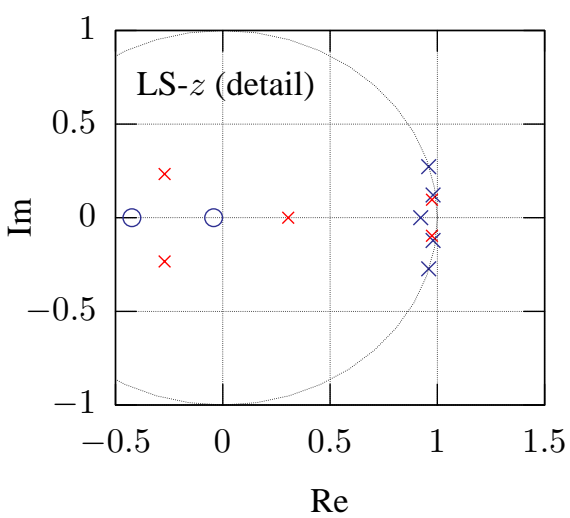

Figure 8. (see Example 4) Results obtained from measurements by invoking the standard least-squares $(L S)$ method with $\tilde{m}=m, T_{a}=10 \mathrm{~ms}$. The representations in terms of $\lambda$ and $\Lambda$ are entitled as " $L S-z$ " and " $L S-q$ " for short

with $\bar{\xi}_{i} \geq \xi_{i}$ ("approximate" compensation as mentioned above takes place for $\bar{\xi}_{i}>$ $\left.\xi_{i}\right)$ and $0<\zeta_{i} \leq 1$. Compensators of this type are usually also referred to as Notch filters. In order to illustrate the quality of the identification result, however, we will exactly cancel out the pole-pair related to the "slow" eigenfrequency via $N_{1}^{\#}(q)$, i.e., set $\bar{\xi}_{1}=\xi_{1}$, see also Figure 10 . The other pole-pair is compensated approximately by setting $\bar{\xi}_{2}=1.5 \xi_{2}$. For both Notch filters of the drive train controller we choose $\zeta_{i}=$ $1, i=1,2$. Finally, as the third part of the controller, in order to achieve steady-state accuracy, we add a transfer function $H$ of PI-type, $H^{\#}(q)=0.08(1+q / 10) / q$, to obtain the controller $R=H N_{1} N_{2}$. The Bode plot of the open-loop transfer function $L=R G$ is also displayed in Figure 10. The BIBO stability of the closed loop $T=$ $L /(1+L)$ is immediately deduced by inspecting the phase angle of $L^{\#}(j \Omega)$ at the cut-off frequency $\Omega_{c}=1.78 \mathrm{rad} / \mathrm{s}$, i.e., $\arg L^{\#}\left(j \Omega_{c}\right)=-120.7 \mathrm{deg}$. Measurement 

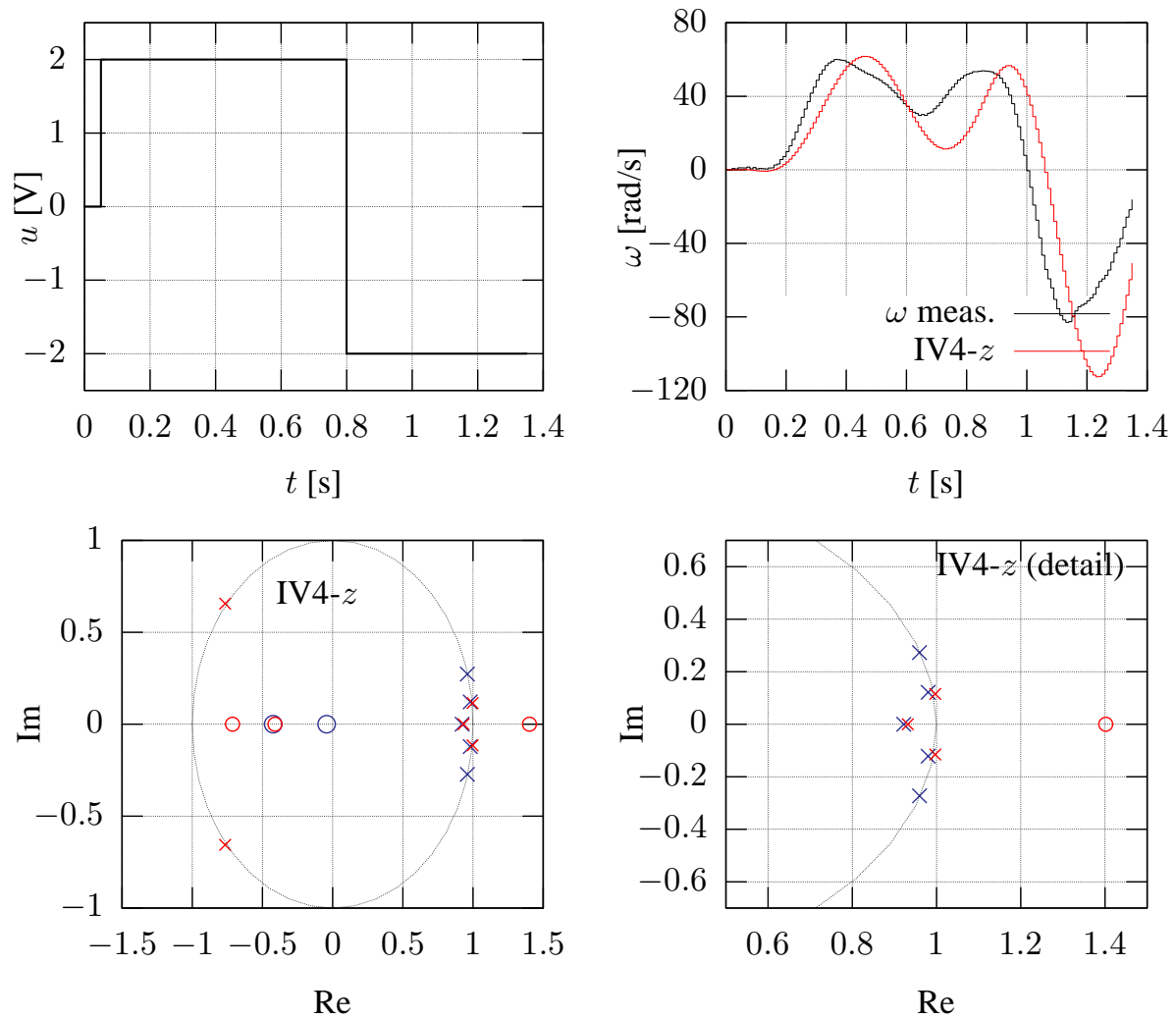

Figure 9. (see Example 6) Results obtained from measurements by invoking the standard instrumental variable method (IV4) implemented in MATLAB with $\tilde{m}=m$, $T_{a}=10 \mathrm{~ms}$

and simulation results of the control loop subject to a step change of the reference angular velocity are finally given in Figure 11 .

Of course, more advanced design techniques, e.g. for decoupling the disturbance and tracking design, might be applied. The primary objective of this simple control example however was to emphasize, by means of measurement and simulation results, the accuracy of the obtained identification result which the control design was based upon. 

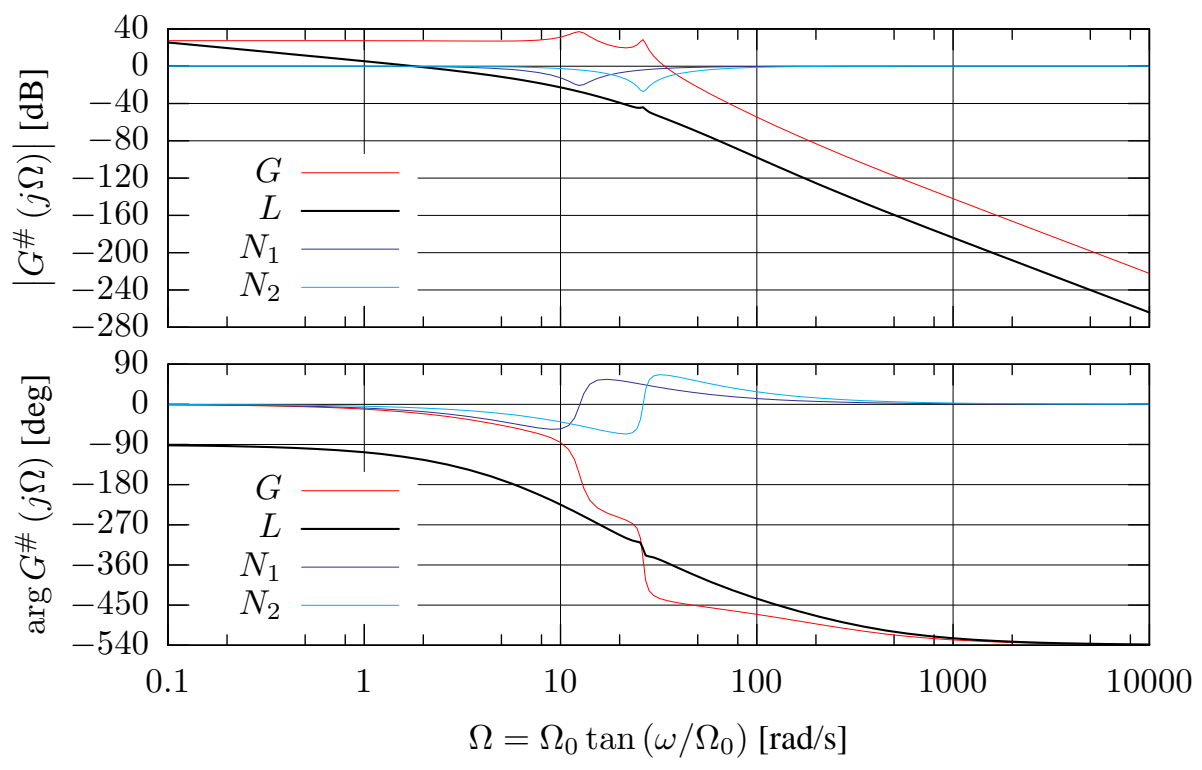

Figure 10. Control application: Bode plots of the identification result $G^{\#}(q)$ due to the measurements of the lab model (see Figure 7), and the plots of the Notch filters $N_{1}, N_{2}$ and the open-loop transfer function $L=R G$

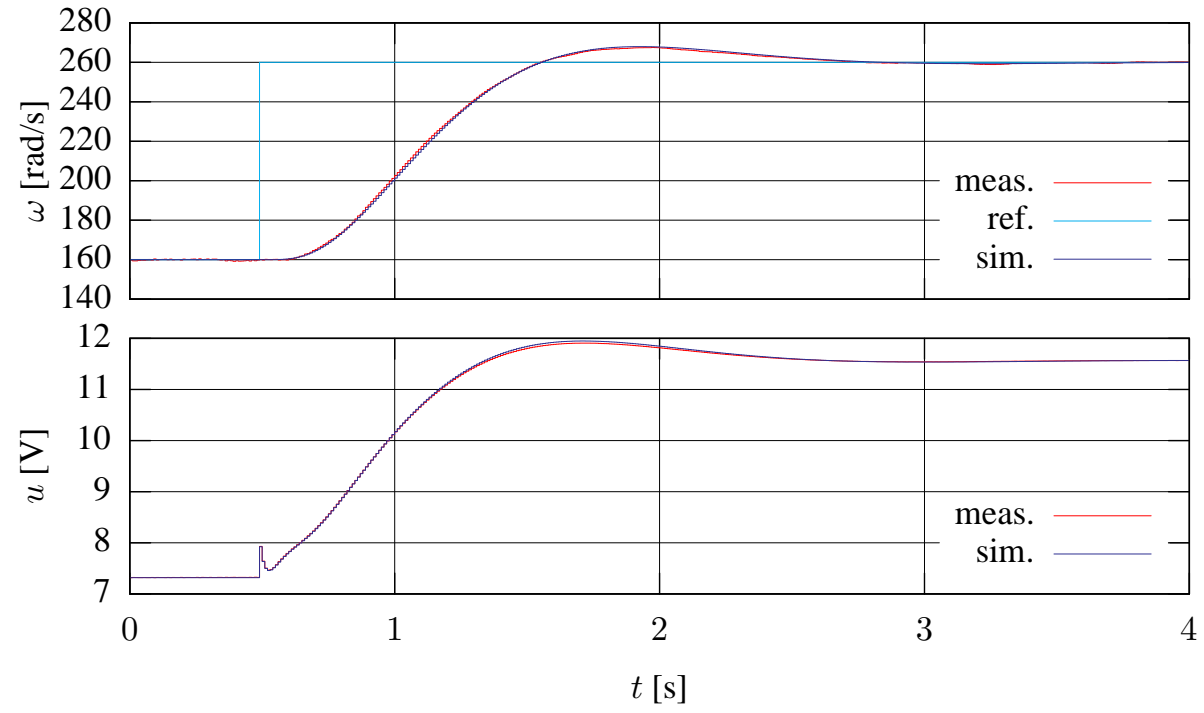

Figure 11. Control application: measurement and simulation results of the control system subject to a step change of the reference angular velocity 


\section{Conclusion}

\subsection{Consequences of the algebraic setting}

The setup of a linear identifier for discrete-time LTI SISO systems, evolving from an algebraic point of view, has been discussed and evaluated on the basis of case studies referring to a fifth-order model of a drive-train. Two different parameterizations have been investigated with regard to their numerical conditioning, with the $q$-domain setting found to exhibiting significant advantages, which become particularly apparent with decreasing sampling times. This effect is observed independently from whether or not the idea of pre-setting certain zeros, which turned out as useful, is applied.

Though this algebraic approach provides promising results, it is worth mentioning that, clearly, the linear identifier cannot incorporate a priori knowledge on stability. More concretely, for the drive-train example, the pole-pair related to the "fast eigenfrequency" is located very close to the stability margin, hence, in presence of noisy signals, the estimated pole-pair might be found to shift beyond the stability margin.

Finally, it should be mentioned that the discussed method provides easy-toimplement on-line identifiers. A computer-algebra implementation of this approach, associated with notes on implementation issues, is available at http://regpro.mechatronik.uni-linz.ac.at

\subsection{Improvement of the mathematical formalism}

Future publications will associate $z$-transforms and module theory ${ }^{2}$ in order to give a more intrinsic algebraic picture of those parametric identification methods and of their extension to multivariable systems. It will thus provide a better understanding of their connections with flatness-based predictive control for discrete-time linear systems ((Fliess et al., 2001; Sira-Ramírez et al., 2004)) and with structural properties as derived from the module-theoretic standpoint (see, e.g., (Bourlès, 2006; Fliess et al., 2001) and the references therein).

\subsection{Nonlinear extension}

A nonlinear extension of our approach is possible via a discrete-time analogue of (Fliess et al., 2008a).

2. Remember that operational calculus and module theory were both already employed in (Fliess et al., 2003; Fliess et al., 2008b). 


\section{References}

Bourlès H., Systèmes linéaires : de la modélisation à la commande, Hermès, Paris, 2006.

Fliess M., "Analyse non standard du bruit", C.R. Acad. Sci. Paris Ser. I, vol. 342, p. 797-802, 2006.

Fliess M., Fuchshumer S., Schlacher K., Sira-Ramírez H., "Discrete-time linear parametric identification: An algebraic approach", Journées Identification et Modélisation Expérimentales - JIME'06, Poitiers, 2006. Available at http://hal.inria.fr/inria-00105673/en/.

Fliess M., Join C., Sira-Ramírez H., "Non-linear estimation is easy", Int. J. Modelling Identification Control, vol. 3, 2008a. Available at http://hal.inria.fr/inria-00158855/en/.

Fliess M., Marquez R., "Une approche intrinsèque de la commande prédictive linéaire discrète", J. europ. syst. automat., vol. 35, p. 127-147, 2001.

Fliess M., Sira-Ramírez H., "On-line discrete-time linear parametric identification: an algebraic approach”, unpublished manuscript, 2002.

Fliess M., Sira-Ramírez H., "An algebraic framework for linear identification”, ESAIM Control Optim. Calc. Variat., vol. 9, p. 151-168, 2003.

Fliess M., Sira-Ramírez H., "Closed-loop parametric identification for continuoustime linear systems", in H. Garnier, L. Wang (eds), Continuous-Time Model Identification from Sampled Data, Springer, Berlin, 2008b. Available at http://hal.inria.fr/inria-00114958/en/.

Fuchshumer S., Algebraic linear identification, modelling, and applications of flatness-based control, Phd thesis, Johannes Kepler Universität, Linz, 2006.

Gausch F., Hofer A., Schlacher K., Digitale Regelkreise, Oldenbourg, Munich, 1991.

Ljung L., System Identification - Theory for the User, $2^{\text {nd }}$ edn., Prentice-Hall, Englewood Cliffs, 1999.

Longchamp R., Commande numérique des systèmes dynamiques, $2^{\text {nd }}$ edn., Presses polytechniques et universitaires romandes, Lausanne, 2006.

Mboup M., "New algebraic estimation techniques in signal processing and control", Fast Estimation and Identification Methods in Control and Signal Processing, $27^{\text {th }}$ Summer School, Laboratoire d'Automatique de Grenoble, Saint-Martin-d'Hères, 2006.

Mboup M., "Parameter estimation via differential algebra and operational calculus", manuscript, 2007. Available at http://hal.inria.fr/inria-00114958/en/.

Middleton R., Goodwin G., Digital Control and Estimation: A Unified Approach, Prentice-Hall, Englewood Cliffs, 1990.

Neves A., Miranda M.D., Mboup M., "Algebraic parameter estimation of damped exponentials", Proc. $15^{\text {th }}$ Europ. Signal Processing Conf. - EUSIPCO 2007, Poznań, 2007. Available at http://hal.inria.fr/inria-00179732/en/.

Sira-Ramírez H., Agrawal S., Differentially Flat Systems, Marcel Dekker, New York, 2004.

Sira-Ramírez H., Fliess M., "On discrete-time uncertain visual based control of planar manipulators: an on-line algebraic identification approach", Proc. $41^{\text {st }}$ IEEE CDC, Las Vegas, 2002.

Söderström T., Stoica P., System Identification, Prentice Hall, New York, 1989. 
Trapero J., Sira-Ramírez H., Battle V., “An algebraic frequency estimator for a biased and noisy sinusoidal signal”, Signal Processing, vol. 87, p. 1188-1201, 2007.

Trapero J., Sira-Ramírez H., Battle V., "On the algebraic identification of the frequencies, amplitudes and phases of two sinusoidal signals from their noisy sums", Int. J. Control, vol. 81, p. 505-516, 2008.

\section{Appendix: the detailed calculations of Section 2}

The following calculations are given to retrace the appearance of [5] by taking $\bar{n} \geq n+1$ derivatives on [3] w.r.t. $z$. Let $(\cdot)^{(j)}=(\partial / \partial z)^{j}(\cdot)$, and notice that

$$
z^{j} f_{z}^{(j)} \bullet(-1)^{j}\left(\left(\prod_{s=0}^{j-1}(k+s)\right) f_{k}\right)
$$

Then, taking the derivatives on both sides of [3] yields $\left(A y_{z}\right)^{(\bar{n})}=\left(B u_{z}\right)^{(\bar{n})}$, and, by virtue of Leibniz' product rule,

$$
\sum_{j=0}^{n}\left(\begin{array}{c}
\bar{n} \\
j
\end{array}\right) A^{(j)} y_{z}^{(\bar{n}-j)}=\sum_{j=0}^{m}\left(\begin{array}{c}
\bar{n} \\
j
\end{array}\right) B^{(j)} u_{z}^{(\bar{n}-j)}
$$

noticing that $A^{(j)}(z)=0, j>n$, and $B^{(j)}(z)=0, j>m$. With $A^{(j)}=$ $\sum_{s=j}^{n} \frac{s !}{(s-j) !} a_{s} z^{s-j}$ and $B^{(j)}=\sum_{s=j}^{m} \frac{s !}{(s-j) !} b_{s} z^{s-j}$, Equation [19] takes the form

$$
\bar{n} ! \sum_{j=0}^{n} \frac{z^{-j} y_{z}^{(\bar{n}-j)}}{(\bar{n}-j) !} \sum_{s=j}^{n}\left(\begin{array}{l}
s \\
j
\end{array}\right) a_{s} z^{s}=\bar{n} ! \sum_{j=0}^{m} \frac{z^{-j} u_{z}^{(\bar{n}-j)}}{(\bar{n}-j) !} \sum_{s=j}^{m}\left(\begin{array}{l}
s \\
j
\end{array}\right) b_{s} z^{s}
$$

To further proceed with [20], and, in particular, in view of facilitating the transformation back to the discrete-time domain, let $F_{z}^{(j)} \doteq z^{j} f_{z}^{(j)} / j !,\left(F_{z},\left(f_{k}\right)\right) \in$ $\left\{\left(Y_{z},\left(y_{k}\right)\right),\left(U_{z},\left(u_{k}\right)\right)\right\}$. Notice that $F_{z}^{(j)} \bullet \multimap \frac{(-1)^{j}}{j !}\left(\left(\prod_{s=0}^{j-1}(k+s)\right) f_{k}\right)$ by [18]. Multiply both sides of Equation [20] by $z^{\bar{n}-n}$. Then, by re-arrangement of the sums for collecting the parameters $a_{i}, i=0, \ldots, n-1$ (notice that $a_{n}=1$ by [1]), and $b_{i}$, $i=0, \ldots, m$, we obtain

$$
\begin{aligned}
& \sum_{i=0}^{n-1} z^{i-n}\left(\bar{n} ! \sum_{j=0}^{i}\left(\begin{array}{l}
i \\
j
\end{array}\right) Y_{z}^{(\bar{n}-j)}\right) a_{i}- \\
& -\sum_{i=0}^{m} z^{i-n}\left(\bar{n} ! \sum_{j=0}^{i}\left(\begin{array}{l}
i \\
j
\end{array}\right) U_{z}^{(\bar{n}-j)}\right) b_{i}=-\bar{n} ! \sum_{j=0}^{n}\left(\begin{array}{l}
n \\
j
\end{array}\right) Y_{z}^{(\bar{n}-j)}
\end{aligned}
$$

Before proceeding with [21], first notice that the identity

$$
\sum_{j=0}^{i}\left(\begin{array}{l}
i \\
j
\end{array}\right) \frac{(-1)^{-j} \bar{n} !}{(\bar{n}-j) !} \prod_{s=0}^{i-j-1}(k-n+\bar{n}+s)=\prod_{s=0}^{i-1}(k-n+s)
$$


holds (with $\bar{n}$ involved in the left-hand side cancelling out). Then, re-sorting to the expressions of [21] associated to the parameters $\left\{a_{i}, b_{i}\right\}$, we have

$$
\begin{aligned}
& z^{i-n} \bar{n} ! \sum_{j=0}^{i}\left(\begin{array}{l}
i \\
j
\end{array}\right) F_{z}^{(\bar{n}-j)} \bullet \\
& (-1)^{\bar{n}}\left(\prod_{s=0}^{i-1}(k-n+s)\right)\left(\prod_{s=i}^{\bar{n}-1}(k-n+s)\right) f_{k-n+i}=(-1)^{\bar{n}} \chi(k) f_{k-n+i}
\end{aligned}
$$

and, hence, the validity of [5]. 